\title{
Sinus lifts failure resulting in chronic sinusitis
}

\begin{abstract}
The article reports on a case of sinus infection management after sinus lift operation. Patient presented to dental clinic with edentulous maxilla for teeth reconstruction with implant supported dental prosthesis. Computer tomography scan has been performed to evaluate alveolar bone property for implantation. As the bone height on both sides was approximately $2 \mathrm{~mm}$, which is inappropriate for implantation, sinus lift procedure was done. After ten days post surgery the patient complained of febrile temperature, pain in left maxillary region, breathing difficulties and purulent discharge from left nostril. Medicinal and surgical treatment process in otorhinolaringological clinic, discussion on possible inflammatory reaction causes and new implantation possibilities are described in the article.
\end{abstract}

Volume 8 Issue 6 - 2017

\author{
Karina Rapsa \\ Riga Stradins University, Latvia
}

Correspondence: Karina Rapsa, Riga Stradins University, Uzvaras prospekts 17, Baloži, Latvia, Tel +37|26575574, Email jelagovakarina@gmail.com

Received:September 12, 2017 | Published: October 20, 2017

Keywords: sinus lift, maxillary sinus floor augmentation, rhinosinusitis

\section{Introduction}

Insufficient bone quality and quantity in posterior maxilla is a common clinical state, which leads to further prosthetic surgery difficulties. It occurs on account of several causes, most frequently due to the pneumatization of sinus subsequent to the tooth loss and the concomitant excessive alveolar resorption. ${ }^{1}$ Depleted alveolar bone is insufficient to host implants of $10 \mathrm{~mm}$ in length and $3-4 \mathrm{~mm}$ in diameter, which are minimum requirements to allow bone-demanded bilateral implant placement in posterior maxilla. Therefore, maxillary sinus floor augmentation has to be done to provide the foundation for consequent implantation. ${ }^{2}$ The sinus lift procedure is a technique of bone reconstruction of the depleted maxillary sinus floor. It is one of the primary surgical options allowing placement of dental implants in the posterior maxilla. Multiple studies have shown that autogenous bone, allogeneic bone, and xenogenic bone graft materials work well along the sinus floor. ${ }^{3}$ The objective of this article is to present a clinical case of bilateral maxillary sinus lift with a year of follow up. Postoperative complications, which presented as a chronic maxillary sinusitis and complete osteograft material failure with nasal discharge and remaining material resorption resulted in oroantral communication on left side. As a result of this processes planned implantation cannot be performed. In a discussion possible causes of complications and options of other methods of teeth prosthetics for this particular patient are discussed.

\section{Case report description}

Data from the patients' anamnesis before she presented to the ENT specialist in the clinic. 52year-old women with edentulous maxilla, presented to dental clinic for prosthetic restorations with implant supported dental prosthesis. Radiologic investigations, including orthopantomography (OPG) and cone-beam computed tomography (CT) were performed to evaluate implantation perspectives. CT revealed bilaterally depleted alveolar bone: $2,21 \mathrm{~mm}$ high on right side and $1,2 \mathrm{~mm}$ high on the left side, which was insufficient for implantation (Figure 1). Therefore, bilateral sinus lift operation was performed. Right maxillary floor augmentation with autogenous bone, left side sinus lift using osteograft material was performed. After ten days post surgery the patient complained of febrile temperature, pain in left maxillary region, breathing difficulties and purulent discharge from left nostril. There were no complaints on right side. She also mentioned, that it is the first such an episode and she never had any rhinosinusitis episodes before. The preoperative CT scan also show healthy maxillary sinus without any inflammatory changes in it, though, other sinuses have not been visualized on preoperative CT scan. According to the patients' complaints, she was prescribed oneweek therapy with Amoxicillin/Clavulanate 500/125 mg orally twice a day. Three months later on the control visit the patient felt absolutely well. Four and a half months after operation the patient presented to ENT specialist due to tightness under the left eye, pain in a forehead and massive purulent nasal discharge including grafting material fragments during the past one and a half months. Objectively mucous membrane of the left nasal pathway was hyperemic, swollen and edematous. Left pathway was full of purulent discharges. The CT scan showed mucosal hyperplasia of a left side maxillary, ethmoidal and frontal sinus (Figure $2 \& 3$ ). Moreover, bone perforation in maxillary sinus floor was visualized on CT scan (Figure 4). This time ten-day antibiotic course with Clindamycin $300 \mathrm{mg}$ three times a day orally was prescribed. Functional Endoscopic Sinus Surgery (FESS) was performed after inflammation decreased to review maxillary sinus and anterior ethmoid cells. $0 \mathrm{o}$ and $30 \mathrm{o}$ rigid endoscopes have been used to visualize the sinus floor. The bone perforation in left maxillary sinus floor covered with mucosa flap from the maxillary and an oral side was detected, all the grafting material was reabsorbed and passed out with the purulent nasal discharge. No grafting material remains were detected on endoscopic examination. Consequently, planned teeth implantation in left posterior maxilla is not possible and a repeated sinus lift procedure is uncertain as well, therefore, other prosthetics method options should be reviewed. After two months on control visit patient did not have any complaints from the nasal symptoms.

\section{Discussion}

Teeth loss provides either functional or aesthetic problems to the patients and tooth implantation is one of the best solutions of this condition. The candidates for implantation procedure must have sufficient bone height and width to support the artificial tooth crown. ${ }^{4}$ According to the literature alveolar bone height must be at least than $10 \mathrm{~mm}$ and the width not less than $4 \mathrm{~mm} .^{5}$ To evaluate remaining alveolar bone height the panoramic radiographic investigation or cone-beam computed tomography investigation should be done. ${ }^{6}$

Implant treatments of the edentulous maxilla occasionally meet with problems due to the lack of bone volume. The most common cause of maxillary sinus floor atrophy is a loss of teeth. Following tooth extraction, as much as $40-60 \%$ ridge resorption can occur within 1-3years. Being edentulous for a prolonged period of time, the 
bone starts to deteriorate, resulting in a lack of bone height to sustain a dental implant. In such cases the sinus lift procedure is performed by placement of the graft material into the maxillary sinus cavity, to prepare the bone for teeth implantation. ${ }^{7}$ Patient identification and correct preparation for sinus lift procedure is of a great importance. For achievement of better results multidisciplinary approach is needed. Othorinolaringologist's consultation is needed before sinus lift procedure to evaluate if patients' sinus health condition is appropriate. Maxillofacial surgeon is responsible for surgery technique and a prosthetist for further implantation strategy. ${ }^{8}$ Implants can be placed simultaneously or as a secondary procedure after graft consolidation. ${ }^{9}$ There are several operation techniques and a variety of grafting materials available such as: autogenous graft (patient's own tissue), allograft (bone from the individuals of the same species), xenografts (bone from other species) and synthetic materials (hydroxyapatite, metals, corals and plastics). Though, nowadays, autogenous bone is the most widely used material for sinus augmentation. It is explained by better osteogenic capacity and a minimal risk of tissue rejection, comparing to other grafting materials. ${ }^{10}$ In a reported case for right maxillary sinus floor augmentation autogenous bone fixed with titanium screws has been used and a synthetic material Osteo Graft alloplast, which is low-density hydroxyapatite, has been used for left side sinus floor augmentation. ${ }^{11}$ Alloplastic grafts have advantages as absence of immunological reaction and toxic effects, which minimize risk of adverse reactions. ${ }^{12}$

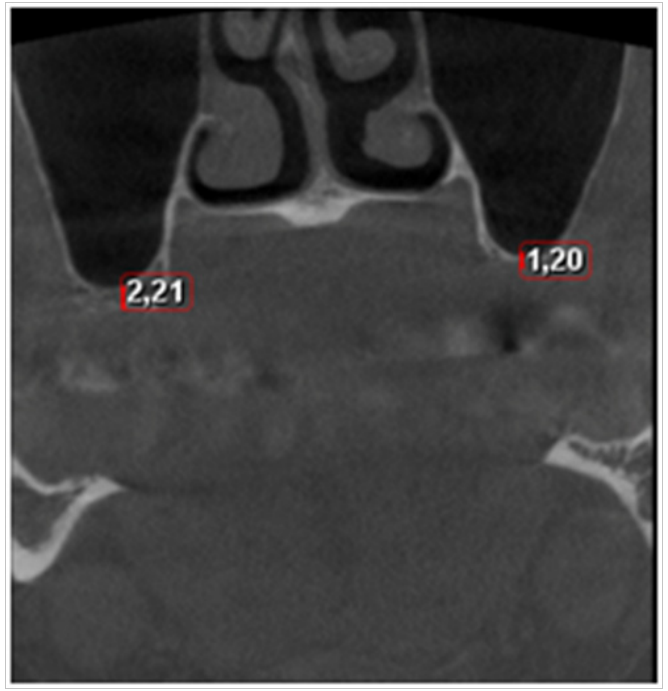

Figure I Coronal views on cone-beam computered tomography scan: Both maxillary sinuses without pathological findings. Depleted alveolar bone 2,2 I $\mathrm{mm}$ high on the right side and I,2 $\mathrm{mm}$ high on the left side.

Although, complications associated with maxillary sinus augmentation are uncommon, in approximately $2-5 \%$, the reported case proves the possibility of their occurrence. All the complications are divided into 4 groups, according to the period they occur: intraoperative, early postoperative, delayed postoperative and late. Intraoperative complications include Schneiderian membrane perforation (the most common), fracture of the residual alveolar ridge, and obstruction of the maxillary ostium, hemorrhage and damage to adjacent dentition. Early postoperative complications occur within three weeks from surgery and consist of: wound dehiscence, infection, graft loss, exposure of barrier membrane. Delayed postoperative complications mainly occur after weeks from the surgery and include: graft loss, implant failure, implant migration, oroantral fistula, chronic pain, chronic sinus disease. The last group are late complications, which occur due to strategy of improperly treated maxillary sinusitis: intracranial and/or intraorbital complications, abscess, blindness and aspergillosis. ${ }^{13,14}$ In infectious complications, treatment depends on spread of the infection. If graft infection is well contained under the Schneiderian membrane its management using only pharmacological therapy is possible. In cases of graft persistence under sinus membrane and symptom persistence longer than three weeks graft removal by oral access combined with pharmacological therapy is indicated? If the Schneiderian membrane is perforated and graft material loss inside the sinus is present than functional endoscopic sinus surgery along with graft material removal is indicated. ${ }^{15}$

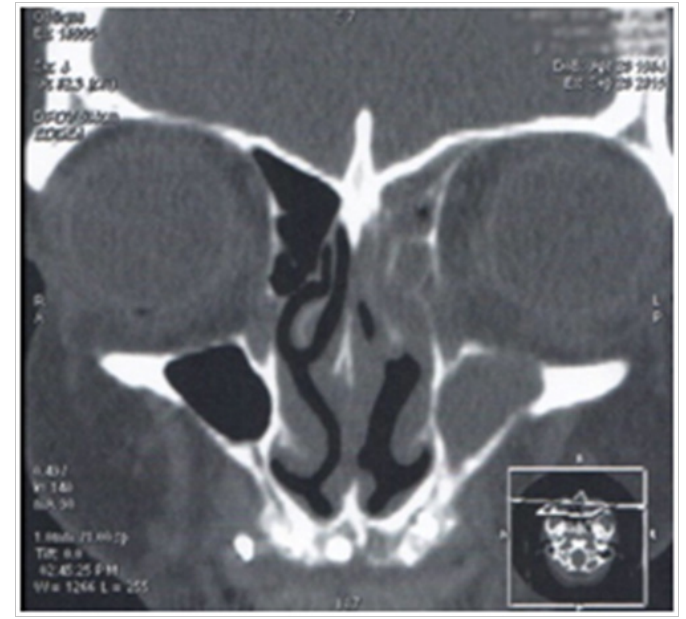

Figure 2 Coronal view of paranasal sinuses on computered tomography scan: Left maxillary sinus and ethmoidal cells filled with inflammatory content.

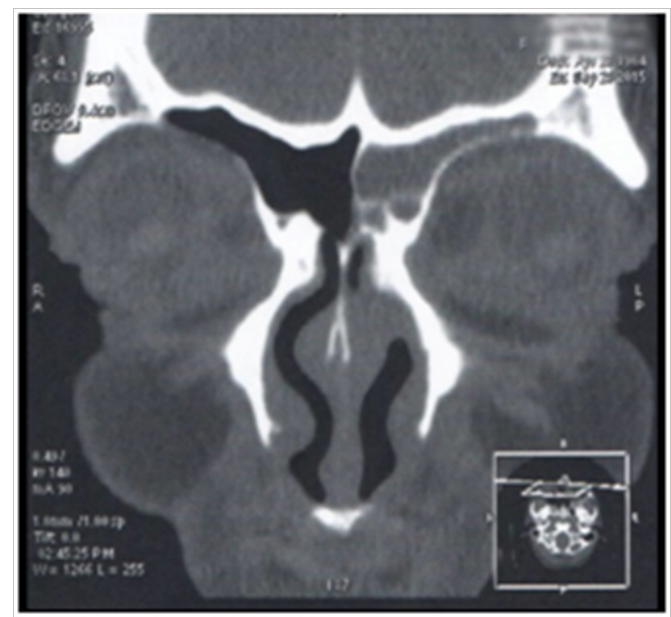

Figure 3 Coronal view on computered tomography scan: Left frontal sinus filled with inflammatory content.

In the presented case, as described before, postoperative sinusitis is developed. As mentioned, women had severe maxillary sinusitis with massive purulent nasal discharge and a pass-out of the grafting material while blowing the nose. It can be proposed, that perforation of Schneiderian membrane occurred and lead to displacement of augmentation material to the sinus and following chronic infection process in a maxillary sinus. According to literature data, if the graft is not contained under the sinus membrane and loss of the graft material into the sinus is present, and symptoms still persist after extended antibiotic therapy, a surgical approach by endoscopic sinus surgery and removal of bone graft is a mandatory way of treatment. ${ }^{16}$ Therefore, functional endoscopic sinus surgery was performed to this 
patient followed by three-day antibiotic therapy with ceftriaxone $2,0 \mathrm{~g}$. intravenously once a day in clinic and two-week antibiotic course with Cefadroxil 500mg orally three times daily. The patient was kept under otorhinolaringologist observation for 3 months. During that period she had neither relapse episodes nor other complaints.

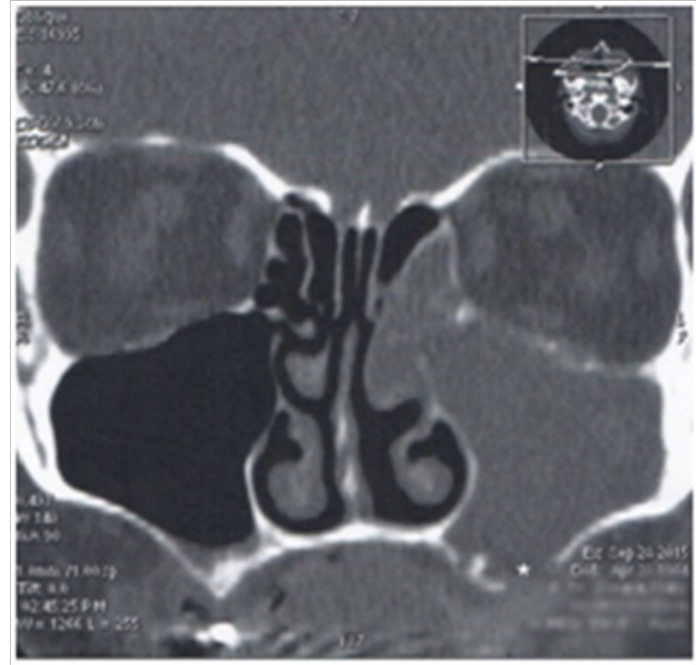

Figure 4 Coronal view on comutered tomography scan: Left maxillaty sinus opacification and blockage of sinus ostium. Bone defect $(*)$ in maxillary sinus floor.

After complete recovery question about further prosthetics plan becomes up front. As the sinus lift of the left maxillary sinus floor failed and resulted in chronic sinusitis. Therefore, another sinus lift procedure could not be performed, cause chronic sinusitis in anamnesis is a contraindication for sinus lift. $^{17}$ Other decision for teeth loss management could be teeth implantation on the right side and anterior maxilla and removable partial denture on the left side of maxilla. Obviously, the removable dentures are not as functionally stable and comfortable as implants, but in this case it is an option to reach moderate function and better cosmetic result. ${ }^{17}$

\section{Conclusion}

Although, sinus lift complications are not frequent, this case shows the severe results of their appearance. So those, the patient selection for procedure, appropriate preoperative investigations and procedure planning take a big part in an outcome of procedure. All indications and contraindication to procedure should be taken into consideration, the procedure should be performed according to treatment protocol and all sterility measures. Appropriate postoperative treatment should be done and postoperative recommendations should be explained to patient.

In case of this patient, despite all things mentioned above, sinus lift procedure failed and embarrassed further process of prosthetic. In such a young patient with teeth loss, other teeth-replacing plan should be developed, to ensure the better functioning and quality of life.

\section{Conflicts of interest}

The authors state no conflict of interest.

\section{Acknowledgments}

None.

\section{Funding}

None.

\section{References}

1. Dogan D, Figel Cizmeci S, Zafer OP. Dental Implants in Posterior Maxilla: Diagnostic and Treatment Aspects. International Journal of Dentistry. 2012.

2. Bernhard P, Werner Z, Georg W, et al. Department of Restorative Dentistry, King's College London, To Graft or Not to Graft? EvidenceBased Guide to Decision Making in Oral Bone Graft Surgery. London, UK. 2012

3. Kademani D. Atlas of Oral and Maxillofacial surgery. The Maxillary Sinus Lift. Elsevier. 2015. p.199-209.

4. http://www.webdental.com/profiles/blogs/types-of-maxillary-sinus

5. William VG, Niklaus PL, Maurizio ST. Osteology Guidelines for Oral \& Maxillofacial Regeneration. In: Gustavo Avil-Ortiz \& Pablo GalindoMoreno (Eds.), Maxillary sinus floor elevation. London: Quintessence Publishing Co; UK. 2014. p.247-259.

6. William V, Niklaus P, Maurizio ST. Osteology Guidelines for Oral \& Maxillofacial Regeneration. In: Erika Benavides \& Hector FR (Eds.), Imaging Technologies to Assess Oral Soft and Hard Tissue regeneration. London: Quintessence Publishing Co; UK. 2014. p.145-158.

7. Karl-Erik Kahnbergs. Bone Grafting Techniques for Maxillary Implants. Inlay Bone Grafting. Blackwell Munksgaard, UK. 2015. p.33-56.

8. Ilze Dobele. Summary of Doctoral Thesis for obtaining the degree of a Doctor of Medicine. Rhinosinusitis Risk Factors in Dentistry-Clinical Aspects. Riga Stradins University, Latvia. 2016.

9. Ole TJ. The sinus bone graft. In: Vincent B, et al. (Eds.), Complications of Maxillary Sinus Augmentation. Denver, Colorado: Quintessence Publishing Co, USA. 1999. p.201-208.

10. Karl-Erik Kahnbergs. Bone Grafting Techniques for Maxillary Implants. Biological Principles of Bone. Blackwell Munksgaard, UK. 2005. p.1-4.

11. Tulsa Dental Specialists. OsteoGRAFT/LD-300. Low Density Hydroxylapatite (ALLOPLAST). Densply International. In: Khoury F \& Antoun H (Eds.), Bone Augmentation in Oral Implantology. Bone Substitutes. Quintessence. 2007.

12. Ole TJ. The sinus bone graft. In: Vincent B, et al. (Eds.), Complications of Maxillary Sinus Augmentation. Denver, Colorado: Quintessence Publishing Co, USA. 1999. p.201-208.

13. Tiziano T, Massimo Del F, Roberto W, et al. Maxillary Sinus Surgery and Alternatives in Treatment. In: Testori, et al. (Eds.), Complications: diagnosis and management. Quintessence Publishing Co, Germany. 2009. p.311-324.

14. Chiapasco M, Felisati G, Maccari A, et al. The Management of Complications Following Displacement of Oral Implants in the Paranasal Sinuses: a Multicenter Clinical Report and Proposed Treatment Protocolos. Int J Oral Maxillofac Surg. 2009;38(12):1273-1278.

15. Fouad K, Hadi A, Patrick M. With contributions. In: Bessade et al. (Eds.), Bone augmentation in oral implantology. Arndt Happe and Fouad Khoury. Complications and risk factors in bone fragting procedures. Quintessence Publishing Co; Germany. 2007. p.405-428.

16. Kademani D. Atlas of Oral and Maxillofacial surgery. The Maxillary Sinus Lift. Elsevier. 2015. p.199-209.

17. Carr Alan B. McCracken's Removable Partial Prosthodontics. In: Alan B, et al. (Eds.), Removable Partial Denture Considerations in Maxillofacial Prosthetics. Elsevier. 2016. p.315-336. 